\title{
The EfFect of Gallium Aluminium ARSENIDE LASER ON FIBROBlast ACTIVITY: An In Vitro Dosimetry Study
}

\begin{abstract}
The effect of different doses of low intensity laser therapy (L.I.L.T.) on human fibroblasts was investigated to determine the optimal dose required to stimulate fibroblast prolfferation. Human SUSAN MARS, ANIL CHUTURGOON, DHAMARAI PILLAY AND MAURICE MARS fibroblasts were cultured in vitro and irradiated with different energy densities of 830nm continuous output infra-red laser using a Gallium Aluminium Arsenide laser. The fibroblasts were irradiated on three consecutive days at energy densities, ranging from 0.2 to $5 \mathrm{J.m}^{2}$, delivered at an average radiant power of $30 \mathrm{~mW}$, and at a constant distance of $1 \mathrm{~cm}$ from the fibroblasts. Fibroblast activity was assessed on the fourth day using a calorimetric MTT (tetrazolium) cleavage assay. There was a significant increase in fibroblast proliferation at laser treatment energy densities of 0.4 $J . \mathrm{cm}^{2}$ and $5 \mathrm{J.cm} \mathrm{cm}^{2}$. Difficulties associated with in vivo and in vitro studies of the effect of laser treatment are discussed.
\end{abstract}

KEY WORDS: LASER THERAPY, FIBROBLASTS, CELL CULTURE, DOSE

\section{INTRODUCTION}

Therapeutic lasers used in medicine can be classified as either high or low energy lasers. High energy lasers provide discretely focused and localised thermal energy to coagulate or vaporise tissue, while low energy "athermic" lasers are used in low intensity laser therapy (L.I.L.T.) which has become a popular form of non-invasive treatment to promote wound healing. (Basford, 1989).

Low intensity laser therapy is thought to provide a biostimulatory effect, enhancing the natural process of wound healing (Brom, 1994). Mester, et al., (1985), demonstrated that laser promoted wound healing by increasing collagen formation, vasodilatation and DNA synthesis. Favourable responses to wound healing have been reported following L.I.L.T. (Mester and Jaszsagi-Nagy, 1973; Dyson \& Young, 1985, 1986; Enwemeka, et al., 1990). Despite a large body of in vitro and in vivo research on the use of LILT, the clinician is faced with the problem that there is no generally agreed or scientifically validated dose for laser therapy in humans (Enwemeka, 1988) and the dose related efficacy of

\section{CORRESPONDENCE} Mrs S Mars

Department of Physiology University of Natal Medical School Pvt Bag 7 Congella 4013

Fax +312604455 e-mailmars@med.und.ac.za
L.I.L.T. remains elusive. (Kitchen \& Partridge, 1991). This is due in part to inadequate reporting of dosage in terms of laser power, area of application, duration and frequency of treatment and the use of different methods, lasers and experimental models. (Baxter, 1994). Further research in the form of thoroughly documented and controlled studies are necessary. (Miller \& Dyson, 1996).

Adequately controlled clinical studies on the efficacy of L.I.L.T. on wound healing are difficult to conduct because of the large number of variables involved in the healing process. Some control can be achieved by studying the components of the healing process in isolation in vitro, for example, the fibroblast which plays an important role in wound healing. The triphasic model of wound healing describes sequential, partially overlapping, inflammatory, proliferative and remodelling phases. (Clark, 1988). During the inflammatory phase, fibronectin and other cytokines recruit fibroblasts to the injured area and encourage fibroplasia. (Silver, 1984). The fibroblasts which proliferate during granulation may transform into actin rich myofibroblasts which have the ability to contract and move over each other, thus contributing to wound contraction. (Clark, 1988; Gabbiani, et al., 1972). Myofibroblasts synthesise collagen which increases the tensile strength of the healing tissue. As fibroblasts are relatively easily grown in cell culture, they make a suitable model for the investigation of the effects of laser therapy.

The majority of studies of L.I.L.T. on fibroblast function have used the Helium
Neon (He-Ne) laser. Following He-Ne laser treatment, fibroblasts have been shown to proliferate (Boulton \& Marshall, 1986), transform into myofibroblasts within 24 hours (PourreauSchneider, et al., 1990) and increase their collagen production (Bosatra, et al., 1984). The most commonly used laser for L.I.L.T in clinical practice is however the infra-red Gallium Aluminium Arsenide (Ga-Al-As) laser. (Baxter, et al., 1991). It is a commercially available, "athermic", diode-based laser emitting light at a wave length of $830 \mathrm{~nm}$ and an average power of $30 \mathrm{~mW}$. Little is known of its effects on fibroblasts as previous studies reporting on the use of Ga-Al-As laser have not specifically looked at its effect on fibroblast proliferation. Young, et al. (1989) treated cultured macrophages with different doses of Ga-Al-As laser, and added the supernatant of the culture medium to a culture of fibroblasts to show that a factor released from treated macrophages can stimulate fibroblast proliferation. While Abergel, et al. (1987) showed that Ga-Al-As laser increased collagen gene expression and Skinner, et al. (1996) demonstrated increased procollagen production by monitoring 3 hydroxyproline synthesis.

The aim of this study was to investigate the effect of infra-red Ga-Al-As laser therapy on a component of the wound healing process, human fibroblast activity, and to determine the optimal dose required to stimulate fibroblast activity in vitro.

\section{METHODS}

Cell Culture. Human fibroblasts were 
obtained from the Department of Virology, University of Natal Medical School and cultured according to the standard laboratory method of Freshnay (1988), in 25ml flasks. Flasks that contained cells with a 20 to $30 \%$ confluency required a routine medium change supplemented with $5 \%$ foetal calf serum (FCS). Commercially available Eagle's Minimum Essential Medium (EMEM) supplemented with Earle's Salts, Hepes, non-essential amino acids and L-glutamine was routinely used. In addition heat inactivated FCS, antibiotics (penicillin, streptomycin and fungizone), Hank's balanced salt solution (HBSS) and trypsin were used.

The cells were routinely passaged as follows:

Cultured flasks containing medium with depleted nutrients were aspirated together with loosely attached cells. These flasks were then rinsed with HBSS $(10 \mathrm{ml})$ at $37 \mathrm{C}$ to remove cellular debris and further washed twice in HBSS. All cell culture procedures were carried out under strict aseptic conditions using a laminar flow.

Cell detachment was detected microscopically or viewed with the naked eye (detached cells gave the medium a cloudy appearance). Five millilitres of culture medium containing 5\% antibiotics was then added and the flasks were returned to the incubator $(37 \mathrm{C})$.

The fibroblasts were confluent after 3 days of subculture. The medium from culture flasks containing confluent fibroblasts was discarded and the cells were washed twice with HBSS.

A $1 \mathrm{ml}$ trypsin solution $(0.25 \%)$ was added to the confluent flasks and trypsin digestion was allowed to proceed for approximately 1 to 5 minutes until microscopic observation showed the cells to have rounded off slightly but not to have become dislodged from the surface. The trypsin was then removed with a sterile Pasteur pipette. Culture medium (1.5ml) containing $10 \%$ FCS was added to the flask and the cells were dispersed in the solution by tapping the flask firmly against the hand. The resultant suspension was repeatedly pipetted with a sterile pipette to separate clumps of cells. To the $0,5 \mathrm{ml}$ cell suspension was added $4 \mathrm{ml}$ of medium for a 1:3 split. Culture flasks $(50 \mathrm{ml})$ were then stoppered and incubated at $37 \mathrm{C}$ without further disturbance until the cells attached to the substrate.

Confluent fibroblast cells were trypsinized and resuspended in culture medium using a haemocytometer, to give a cell count of $11 \times 104$ cells $/ \mathrm{ml}$. This cell suspension was used to fill each of the wells of a 96 well microtiter plate with $100 \mu \mathrm{l}$ of cell suspension per well. Two hundred microlitre culture medium was added to each well. Control wells also contained $100 \mu$ l of cell suspension and $200 \mu \mathrm{l}$ of culture medium. The microtiter plate was incubated for 24 hours at $37 \mathrm{C}$.

Cell Irradiation. Each well was then irradiated three times on consecutive days, i.e. at 24,48 and 72 hours post plating of the fibroblasts. A Ga-Al-As Endolaser 476, infrared (Class3B), continuous, low intensity laser was used. The wavelength was $830 \mathrm{~nm}$ and the average power output of the laser was 30 $\mathrm{mW}$.

The laser probe was swabbed with absolute alcohol and aseptic conditions were strictly adhered to when treating the cell culture.

The laser probe was held perpendicular to, and steady at, the "mouth" of each well at a constant distance of $1 \mathrm{~cm}$ from the fibroblast layer on the base of each well. Each column (2-11) was irradiated with a pre-set dose measured in $\mathrm{J} . \mathrm{cm}^{2}$. Columns 1 and 12 served as controls and were not irradiated. The Endolaser probe is fitted with a target light to clearly indicate the treatment area and protective eye goggles were worn. Each dose was preset on the Endolaser, with the laser unit automatically setting the correct time requirement according to the selected output. A continuous, $100 \%$ output was selected.

Only 1 well was exposed at a time, the other wells were screened with tinfoil to prevent accidental irradiation. Culture medium was renewed daily from each well, and the plate was incubated at $37 \mathrm{C}$. Each of the 3 irradiations was performed at the same time daily. The doses used were $0.2,0.4,0.6,0.8,1,1.5,2.3,3,4$, and $5 \mathrm{~J} . \mathrm{cm}^{2}$ for each column of 8 wells respectively. The doses were calculated on a well area of $0.3318 \mathrm{~cm}^{2}$.

Fibroblast Viability Assay. Cell Viability was measured using the MTT [3-(4.5-dimethylthiazol-2-yl)-2.5diphenyl tetrazolium bromide] reduction assay. (Hanelt, et al., 1994). The principle of this assay is that the tetrazolium salt (yellow) is reduced to a formazan (blue) dye by the succinate dehydrogenase enzymes of the mitochondria of viable cells.

The wells were decanted and $100 \mu$ l of fresh culture medium was added together with $10 \mu \mathrm{l}$ of MTT $(10 \mathrm{l} / 100 \mathrm{l})$ and the plates were further incubated for 4 hours at $37 \mathrm{C}$. Supernatants were then aspirated and $100 \mu 1$ of DMSO (dimethylsulfoxide) was added to each well to solubilise the formazan crystals and incubated for a further hour at 37C. Each well was diluted by a factor of 10 and the optical density of each well was measured spectrophotometrically using a Bio-Rad spectroreader at a wavelength of $595 \mathrm{~nm}$, with a reference wavelength of $630 \mathrm{~nm}$. The spectrophotometric assay was repeated three times for each sample and the average recorded.

The absorbance readings were made with the operators blinded to the dose received by the sample.

\section{RESULTS}

Cell Culture. The fibroblast cells grew rapidly and after three days in culture the cells appeared scattered on the substrate. The cells were monolayered, agranular and were tightly adherent to one another. After a week in culture, cells appeared tightly packed together. Many cells $(+80 \%)$ displayed prominent nuclei, which were observed by phase contrast microscopy (x1600).

Cell Viability. The results of the MTT assay using the 96 well plates are shown in table 1 . The cells were diluted 10 times to ensure that absorbance readings were within the range of the spectrophotometer. Analysis of variance (ANOVA) showed a significant difference between the controls and the treated cells $(\mathrm{F}=$ 2.827 and $\mathrm{P}=0.004)$. Post hoc testing using unpaired $\mathrm{T}$ tests with the Bonferonni correction showed only the doses of $0.4 \mathrm{~J} . \mathrm{cm}^{2}$ and $5 \mathrm{~J} . \mathrm{cm}^{2}$ to differ significantly from the controls. The coefficient of variance ranged from $21.9 \%$ to $39.8 \%$.

\section{DISCUSSION}

The nomenclature used to describe LILT dosage has caused much confusion, and makes interpretation of the literature difficult. Dosage has been reported independently in terms of time (s), power output $(\mathrm{W})$, energy $(\mathrm{J}=\mathrm{W} . \mathrm{s})$, power density $\left(\mathrm{W} . \mathrm{cm}^{2} . \mathrm{s}\right)$ or energy density $\left(\mathrm{J} . \mathrm{cm}^{2}\right)$. Ideally dosage should be described in terms of the total dose of energy supplied per unit area to the tissue, the energy density, expressed in Joules per square centimetre $\left(\mathrm{J} . \mathrm{cm}^{2}\right)$.

To determine energy density, the area of the light beam incident on the surface of the skin or wound (the spot size), the average radiant power of the laser source, the duration of irradiation, the 
depth of the target tissue and the absorbance and scattering characteristics of the tissues through which the light is passing must be known. Comparison of the literature is further complicated by the observation that the results obtained at an energy density produced by a laser of one wavelength, may not be valid or reproducible when using a laser of a different wavelength.

Most previous studies on fibroblast proliferation have used the $\mathrm{He}-\mathrm{Ne}$ laser (632.8nm). (Mester, et al., 1978; Abergel, et al., 1984; Tocco, et al., 1985; Boulton and Marshall, 1986; Hallman, et al., 1988; Pourreau-Schneider, et al., 1990; Zugaro, et al., 1991; Lubart, et al., 1992; Van Brugel \& Bar, 1992). The HeNe laser usually emits a non pulsed continuous waveform. The semiconductor Ga-Al-As laser (830nm) used in this study is currently the most popular in the clinical situation (Baxter, et al., 1991) and may be used in continuous or pulsed mode. The three studies reporting the use of the Ga-Al-As laser investigated its effect on macrophage stimulation of fibroblasts (Young, et al., 1989), on gene expression of collagen formation (Abergel, et al., 1987) and on collagen production and DNA replication (Skinner, et al., 1996). We could find no previous studies on the use of continuous mode Ga-Al-As laser on fibroblasts.

Using the He-Ne laser Van Breugel and Bar (1992), felt that power output as well as irradiation time was important. They found that irradiation times of 0.5 2 minutes to be most stimulatory, and that power outputs of $0.55-5.98 \mathrm{~mW}$ enhanced cell proliferation. Unfortunately the surface area irradiated was not stated, so the energy density is not known. In the present study, the treatment time was calculated for each dose to ensure that the stated energy density was delivered.

Based on $\mathrm{He}-\mathrm{Ne}$ laser wound healing studies, Mester and Jaszsagi-Nagy (1973) stated that the therapeutic treatment window was between energy densities of $1-4 \mathrm{~J} . \mathrm{cm}^{2}$ and that above $4 \mathrm{~J} . \mathrm{cm}^{2}$ a saturation effect occurred. Bolton, et al (1990; 1991) found maximal fibroblast proliferation at energy densities of 2.4 $7.2 \mathrm{~J} . \mathrm{cm}^{2}$. This is in accordance with the Arndt-Schultz Law which states that there is, theoretically, an optimal threshold dose to achieve a biostimulatory effect in the target tissue, but that at a certain point saturation will occur and a plateau effect will occur. (Baxter, 1994). In the present study, no saturation effect was noted and significant differences in fibroblast proliferation were seen at 0.4 and $5 \mathrm{~J} . \mathrm{cm}^{2}$. It is unusual to find reports of fibroblast stimulation at a dose as low as $0.4 \mathrm{~J} . \mathrm{cm}^{2}$. Between energy densities of $0.6 \mathrm{~J} . \mathrm{cm}^{2}$ to $4 \mathrm{~J} . \mathrm{cm}^{2}$ there was no statistical evidence of an increase in cell turnover. At $1 \mathrm{~J} . \mathrm{cm}^{2}$ and $3 \mathrm{~J} . \mathrm{cm}^{2}$ the mean absorbance values noted were less than that of the controls.

There are very few studies to have used a dose as low as $0.4 \mathrm{~J} . \mathrm{cm}^{2}$ and the stimulatory effect at this dose is unusual and requires further investigation. The differences noted in dose response between $\mathrm{He}-\mathrm{Ne}$ and $\mathrm{Ga}-\mathrm{Al}-\mathrm{As}$ may be due to the different wavelength of light utilised or experimental differences.

It could be argued that the assay used to demonstrate cell viability was inappropriate or not sensitive enough for this type of study. It was chosen because laser irradiation causes increased ATP production in the ferric sulphide redox system in the mitochondria and the resultant increase in energy production increases cell function. (Karu, 1987). The MTT assay, which measures the activity of succinate dehydrogenase in the mitochondria has been used successfully in many other cell culture studies and should be sufficiently sensitive to identify changes in cell proliferation. Another possible explanation of the findings may be that the MTT assay should have been delayed until the latent biostimulatory

Table 1. Absorbance readings for each of the 96 wells following MTT assay. Columns 1 and 12 were not lasered and served as controls.

\begin{tabular}{|c|c|c|c|c|c|c|c|c|c|c|c|c|}
\hline \multicolumn{13}{|c|}{ Column } \\
\hline & 1 & 2 & 3 & 4 & 5 & 6 & 7 & 8 & 9 & 10 & 11 & 12 \\
\hline \multicolumn{13}{|c|}{ Dosage J.cm-2 } \\
\hline & 0 & $0.2 \mathrm{~J}$ & $0.4 \mathrm{~J}$ & $0.6 \mathrm{~J}$ & $0.8 \mathrm{~J}$ & $1.0 \mathrm{~J}$ & $1.5 \mathrm{~J}$ & 2.3J & 3J & $4 \mathrm{~J}$ & $5 \mathrm{~J}$ & 0 \\
\hline Row & & & & & & & & & & & & \\
\hline$A$ & 0.71 & 1.00 & 0.77 & 1.06 & 1.09 & 0.66 & 0.96 & 0.63 & 1.05 & 1.25 & 1.29 & 0.93 \\
\hline B & 1.12 & 1.46 & 0.92 & 1.11 & 1.11 & 0.87 & 1.18 & 0.44 & 0.52 & 0.95 & 1.84 & 0.97 \\
\hline C & 0.69 & 0.77 & 1.04 & 1.22 & 0.25 & 0.85 & 0.83 & 1.27 & 0.57 & 0.55 & 1.35 & 0.99 \\
\hline D & 0.61 & 1.44 & 1.71 & 0.96 & 1.16 & 0.44 & 1.27 & 1.04 & 0.42 & 0.95 & 1.66 & 0.9 \\
\hline$E$ & 0.71 & 1.07 & 1.71 & 0.81 & 0.81 & 0.5 & 0.62 & 0.77 & 0.48 & 0.88 & 0.86 & 1.24 \\
\hline $\mathrm{F}$ & 0.62 & 0.87 & 1.04 & 0.94 & 1.06 & 1.11 & 0.31 & 0.65 & 0.58 & 0.72 & 0.53 & 1.03 \\
\hline$G$ & 0.38 & 0.98 & 1.06 & 0.65 & 1.06 & 1.08 & 0.64 & 0.91 & 0.66 & 1.22 & 1.16 & 0.47 \\
\hline $\mathrm{H}$ & 0.73 & 0.72 & 1.05 & 0.68 & 1.05 & 0.56 & 0.63 & 1.11 & 0.97 & 0.77 & 0.92 & 0.82 \\
\hline Avg & 0.70 & 1.04 & 1.16 & 0.93 & 0.95 & 0.76 & 0.81 & 0.85 & 0.66 & 0.91 & 1.20 & 0.92 \\
\hline Std & 0.21 & 0.28 & 0.35 & 0.20 & 0.30 & 0.26 & 0.32 & 0.28 & 0.23 & 0.24 & 0.43 & 0.22 \\
\hline $\mathrm{COV}$ & 29.5 & 26.9 & 30.2 & 21.9 & 31.7 & 33.9 & 39.8 & 32.8 & 35.1 & 26.3 & 35.8 & 23.8 \\
\hline
\end{tabular}


response noted by Rajarathan, et al. (1994) occurred. They noted no differences in proliferation at 72 hours post treatment, but significant changes at 120 hours.

The few clinical studies on non-healing ulcers which report L.I.L.T. dosage in terms of energy density, suggest that energy densities of $4 \mathrm{~J} . \mathrm{cm}^{2}$ (Mester and Mester, 1989), $4.5 \mathrm{~J} . \mathrm{cm}^{2}$ (Khurshudian, 1989) are optimal for promoting healing. This is in keeping with the observations of the in vitro increase in fibroblast proliferation at $5 \mathrm{~J} . \mathrm{cm}^{2}$.

A limitation to this and other in vitro cell culture models is the distance between the probe and the base of the cell culture wells, which in this study was $1 \mathrm{~cm}$. What is not known is the absorbance of light and hence energy by the cell culture medium. As different media may have different absorbencies, dose in cell culture may well then be also medium dependent. Another uninvesti- gated variable is the amount of reflection and refraction of the laser light by the medium. This affects the energy reaching the cells. Although every attempt was made to isolate each well during treatment by screening the other wells with tin foil, some energy may have been transmitted through the plastic of the 96 well plate.

\section{CONCLUSION}

Research into the use of laser in wound healing is beset with many problems. The issues of differences in dose (continuous or pulsed), treatment time, power output, treatment area and treatment depth make comparison of studies difficult. In vitro there are further problems such as the of the lack of the skin layer which will influence delivered dose, no information on the absorbance, refraction, reflection and scattering capacity of culture media, and the attempt to infer from the activity of one cell line in isolation its activity in a multifaceted repair process. In the pre- sent study Ga-Al-As laser delivered in three consecutive daily doses of $0.4 \mathrm{~J} . \mathrm{cm}^{2}$ and $5 \mathrm{~J} . \mathrm{cm}^{2}$ induced increased human fibroblast proliferation in vitro. Review of the literature also suggests that there is sufficient evidence to support the benefits of laser. The exact nature of its beneficial action and the optimal dosages still await elucidation. Further studies investigating laser induced cytokine expression in macrophages and fibroblasts and their effects on the components of the wound healing process are required.

Acknowledgement. This work was funded by the University of Natal, Smith and Nephew Wound Healing Research Group.

This paper was presented in part at the South African Sports Medicine Association Congress - 1997.

Table 1. Absorbance readings for each of the 96 wells following MTT assay. Columns 1 and 12 were not lasered and served as controls.

\section{REFERENCES}

Abergel RP, Lyons RF \& Castel JC. 1987. Biostimulation of wound healing by lasers: experimental approaches in animal models and fibroblast cultures. Journal Dermatological Surgery Oncology, 13:127133.

Abergel RP, Meeker C, Lam T, Dwyer RM, Lesavoy MA \& Vitto J. 1984. Control of connective tissue metabolism by laser. Recent developments and future prospects. Dermatologic Surgery, 11:1142-1150.

Basford JR. 1989. Low-energy laser therapy: controversies and new research findings. Lasers in Surgery and Medicine, 9:1-5.

Baxter GD. 1994. Therapeutic Lasers: Theory and Practice. Churchill Livingstone, London.

Baxter GD, Bell AJ \& Allen JM. 1991. Low level laser therapy. Current clinical practice in Northern Ireland. Physiotherapy, 77: 171178.

Bolton P, Young S \& Dyson M. 1991. Macrophage responsiveness to light therapy with varying power and energy densities. Laser Therapy, 3:105-111.

Bolton P, Young S \& Dyson M. 1990. Macrophage responsiveness to light therapy a dose response study. Laser Therapy, 2:101106.

Bosatra M, Jucci A, Olliano P, Quacci P \& Sacchi S.1984. In vitro fibroblast and dermis fibroblast activation by laser irradiation at low energy. Dermatologica, 168:157-162.

Boulton M \& Marshall J. 1986. He-Ne laser stimulation of human fibroblast proliferation and attachment in vitro. Lasers in Life Sciences, 1:125-134.
Brom B. 1994. Low energy Laser Treatment in General Practice. South African Journal of Family Practice, 15:226-235.

Clark RAF. 1988. Overview and general considerations of wound repair. In Clark RAF, Henson PM, (eds.) The Molecular and Cellular Biology of Wound Repair, pp 3-33. Plenum Press, New York.

Dyson M \& Young S. 1985. The effect of laser therapy on wound contraction. International Congress Laser Medicine Surgery, pp 215-219.

Dyson M \& Young S. 1986. The effect of laser therapy on wound contraction and cellularity in mice. Lasers in Medical Science, 1:125-130.

Enwemeka CS, Rodriquez O \& Gall NG. 1990. Correlative ultrastructural and biomechanical changes induced in regenerating tendons exposed to laser photo-stimulation. Lasers in Surgery and Medicine, Suppl. 2:12.

Enwemeka CS. 1988. Laser biostimulation of healing wounds: Specific effects and mechanisms of action. Journal of Orthopaedics and Sports Physiotherapy, 9:333-338.

Freshnay RI. 1988. Culture of animal cells: a manual of basic techniques. 2nd edition, Liss, New York.

Gabbiani G, Hirschel BJ, Ryan GB, Statkov PR \& Majno G. 1972. Granulation tissue as a contractile organ. A study of the structure and function. Journal of Experimental medicine, 135: 719-734.

Hallman HO, Basford JR, O'Brien JF \& Cummings LA. 1988. Does low energy He$\mathrm{Ne}$ laser irradiation alter in vitro replication of human fibroblasts? Lasers in Surgery and Medicine 8:125-129.
Hanelt M, Gareis M \& Kollarczik B. 1994. Cytotoxicity of mycotoxins evaluated by the MTT cell culture assay. Mycopathologia, 128:167-174.

Karu TI. 1987. Photobiological fundamentals of low-power laser therapy. Journal of Quantum Electronics, 23:1703-1717.

Khurshudian AG. 1989. Use of He-Ca lasers in the complex treatment of suppurative disease in patients with diabetes mellitus. Khirurgiya, 6:38-42.

Kitchen SS \& Partridge CJ. 1991. A review of low level laser therapy. Physiotherapy, 773:161-163.

Lubart R, Wollman Y, Friedmann H, Rochkind S \& Laulicht I. 1992. Effects of visible and near - infrared lasers on cell cultures. Journal of Photochemistry and Photobioliogy, 12:305-310.

Mester E, Jaszsagi \& Nagy E. 1973. Effect of laser radiation on wound healing and collagen synthesis. Studies in Biophysics, 35:227-230.

Mester E, Mester AE \& Mester A. 1985. The biomedical effect of laser application. Lasers Surgery and Medicine, 5:31-39.

Mester AF \& Mester A. 1989. Wound healing. Laser Therapy, 1:7-15.

Mester E, Nagylucskay S, Tisza S \& Mester A. 1978. Stimulation of wound healing by means of laser rays. Acta Chirurgica Academiae Scientificarum Hungericae 19:163-170.

Miller M \& Dyson M. 1996. Principles of wound Care. A Professional Nurse publication. Macmillan Magazines Ltd. London.

Pourreau-Schneider N, Ahmed A, Sondry M, Jacquemier J, Kopp F, Franquin JC \& Martin PM. 1990. Helium - neon laser 
treatment transforms fibroblasts into myofibroblasts. American Journal of Pathology, 137:171-178.

Rajarathan S, Bolton P \& Dyson M. 1994. Macrophage responsiveness to laser therapy with varying pulsing frequencies. Original article, Tissue Repair Research unit, United Medical and Dental Schools, Guy's Hospital Campus, London.

Silver IA. 1984. Oxygen and Tissue repairs. In Ryan TJ (ed.) An environment for healing: the role of occlusion. Royal Society of Medicine International Congress and Symposium Series, 1: 88:18.

Skinner SM, Gage JP, Wilce PA \& Shaw RM. 1996. A preliminary study of the effects of laser radiation on collagen metabolism in cell culture. Australian Dental Journal, 41:188-192, 1996.

Tocco G, Le Borgue de Kaovel C \& Aubert C. 1985. He Ne and I.R. mid laser influences in skin cells in vitro; preliminary results. Proceedings of the International Congress of Lasers in Medicine and Surgery, 175-182.

Van Breugel HH \& Bar PR. 1992. Power density and exposure time of He-Ne laser irradiation are more important than total energy close in photo-biomodulation of human fibroblasts in vitro. Lasers in Surgery and Medicine, 12:528-537.

Young S, Bolton P, Dyson M, Harvey W \& Diamantopoulis C. 1989. Macrophage responsiveness to light therapy. Lasers in Surgery and Medicine, 9:497-505.

Zugaro A, Rizzo GL, Piccinni M, Continenza MA \& Ricciardi G. 1991. Application of infrared lasers on in vitro culture of fibroblasts: effects of the duration of treatment parameter. Annali Italiani di Chirurgia, 62:577-580.

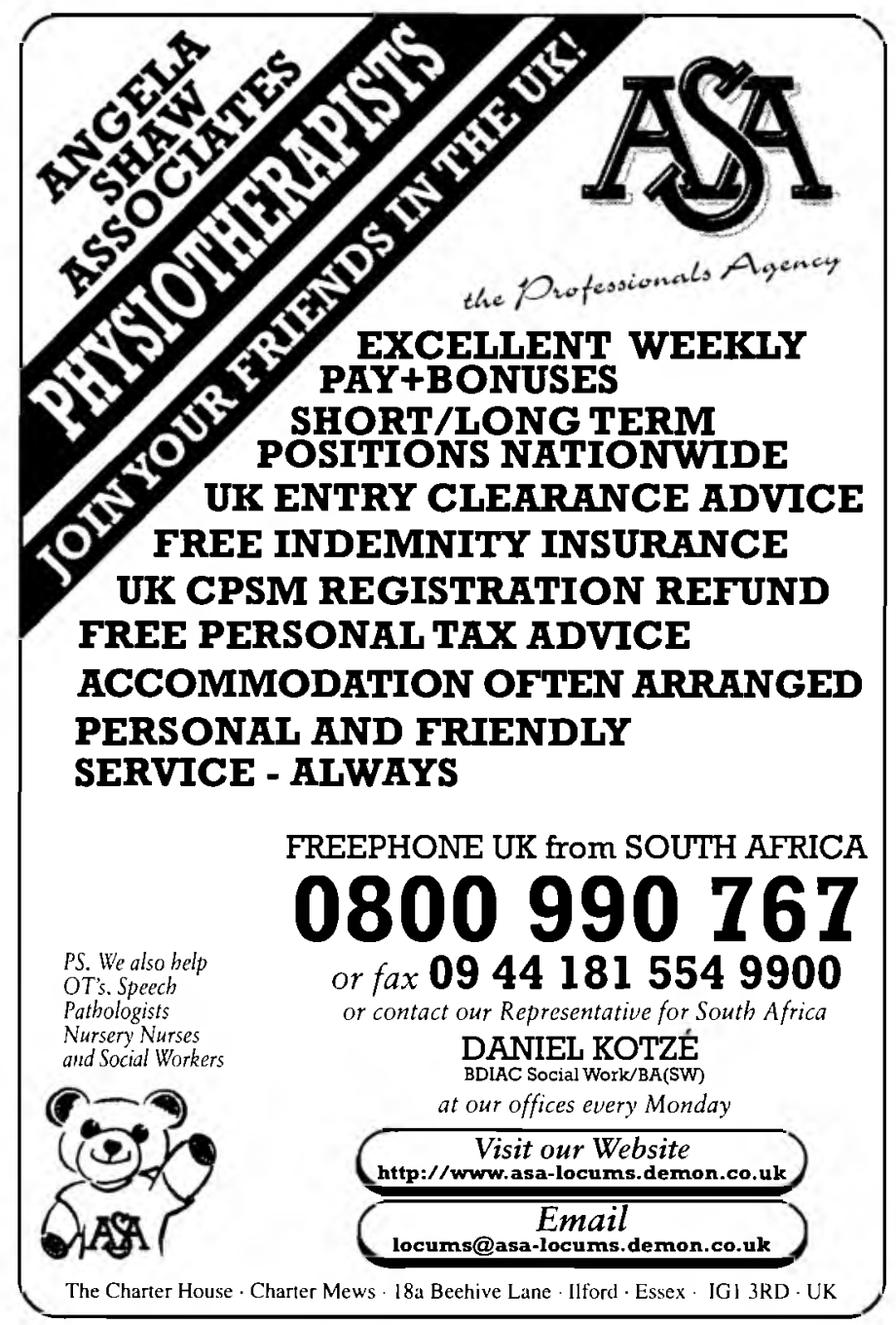

